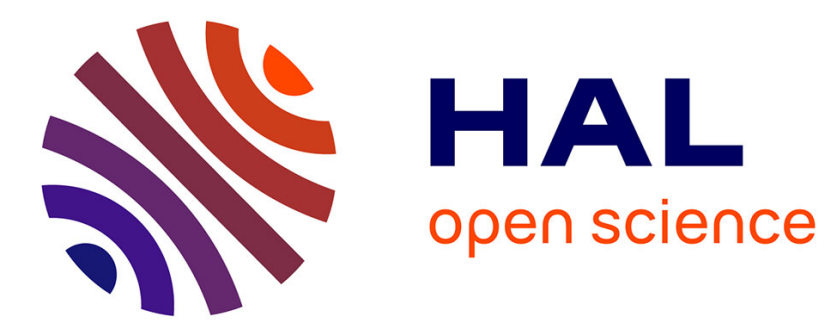

\title{
Effect of size polydispersity versus particle shape in dense granular media
}

Duc-Hanh Nguyen, Émilien Azéma, Farhang Radjai, Philippe Sornay

\section{To cite this version:}

Duc-Hanh Nguyen, Émilien Azéma, Farhang Radjai, Philippe Sornay. Effect of size polydispersity versus particle shape in dense granular media. Physical Review E: Statistical, Nonlinear, and Soft Matter Physics, 2014, 86 (041301), pp.1-15. 10.1103/PhysRevE.90.012202 . hal-01059980

\section{HAL Id: hal-01059980 https://hal.science/hal-01059980}

Submitted on 2 Sep 2014

HAL is a multi-disciplinary open access archive for the deposit and dissemination of scientific research documents, whether they are published or not. The documents may come from teaching and research institutions in France or abroad, or from public or private research centers.
L'archive ouverte pluridisciplinaire HAL, est destinée au dépôt et à la diffusion de documents scientifiques de niveau recherche, publiés ou non, émanant des établissements d'enseignement et de recherche français ou étrangers, des laboratoires publics ou privés. 


\title{
Effect of size polydispersity versus particle shape in dense granular media
}

\author{
Duc-Hanh Nguyen,,${ }^{1,2, *}$ Emilien Azéma, ${ }^{1, \dagger}$ Farhang Radjai,,${ }^{1,}$ and Philippe Sornay ${ }^{2, \S}$ \\ ${ }^{1}$ Université Montpellier 2, CNRS, LMGC, Place Eugène Bataillon, 34095 Montpellier, France \\ ${ }^{2}$ CEA, DEN, DEC, SPUA, LCU, F-13108 Saint Paul lez Durance, France
}

(Received 27 January 2014; published 21 July 2014)

\begin{abstract}
We present a detailed analysis of the morphology of granular systems composed of frictionless pentagonal particles by varying systematically both the size span and particle shape irregularity, which represent two polydispersity parameters of the system. The microstructure is characterized in terms of various statistical descriptors such as global and local packing fractions, radial distribution functions, coordination number, and fraction of floating particles. We find that the packing fraction increases with the two parameters of polydispersity, but the effect of shape polydispersity for all the investigated structural properties is significant only at low size polydispersity where the positional and/or orientational ordering of the particles prevail. We focus in more detail on the class of side/side contacts, which is the interesting feature of our system as compared to a packing of disks. We show that the proportion of such contacts has weak dependence on the polydispersity parameters. The sideside contacts do not percolate but they define clusters of increasing size as a function of size polydispersity and decreasing size as a function of shape polydispersity. The clusters have anisotropic shapes but with a decreasing aspect ratio as polydispersity increases. This feature is argued to be a consequence of strong force chains (forces above the mean), which are mainly captured by side-side contacts. Finally, the force transmission is intrinsically multiscale, with a mean force increasing linearly with particle size.
\end{abstract}

DOI: 10.1103/PhysRevE.90.012202

PACS number(s): 45.70.Cc, 61.43.-j

\section{INTRODUCTION}

Polydispersity means "several sources of dispersion," which is conventionally summarized by particle size distribution in the case of granular media. There are, however, other origins of dispersion in granular materials, such as particle shape and density. Shape polydispersity can be defined as dispersion of a parameter describing the shape of the particles. Major shape characteristics are elongation, angularity, and convexity. Shape polydispersity and size polydispersity are widespread in nature. In many industrial processes involving granular materials, particle size, and shape need to be optimized for improved performance [1-4]. Polydispersity is also a basic feature of soft matter systems such as colloids, polymers, and liquid crystals [5-9].

Due to its profound effects on the structure of granular systems, the analysis of polydispersity represents a considerable challenge to theory, simulation, and experiment. For monosized particles, systematic studies of particle shape dependence have been recently reported for angular [10-14], elongated [15-24], platy [25], complex shape [26,27], and nonconvex shapes $[28,29]$ as well as for "superballs" [30].

Shape characteristics strongly affect the structure of dense packings as well as their rheology. Among others, an interesting finding evidenced by recent studies is the nonmonotonic relation between the aspherity of the particles (i.e., degree of distortion from a perfectly circular or spherical shape) and the packing fraction [15,30-32]. However, the particles in naturally occurring granular materials have mostly irregular shapes. This irregularity may often be described as a secondorder shape parameter, i.e., as degree of variability around

\footnotetext{
*duc-hanh.nguyen@univ-montp2.fr

†emilien.azema@univ-montp2.fr

${ }^{\ddagger}$ franck.radjai@univ-montp2.fr

§philippe.sornay@cea.fr
}

a well-defined shape. To our best knowledge, no studies have been reported on the effect of polydispersity parameters pertaining to the shapes of the particles.

The effect of particle size distribution on the structure of granular materials has been a subject of great interest [3,4,33-40]. However, most investigations have mainly dealt with packing fraction. For example, bidisperse systems, as mixtures of particles of two different sizes, have been modeled in this regard [38,41-44]. For a given size ratio, the packing fraction first increases with the proportion of small particles, but begins to decline back to that of a monodisperse packing at still larger proportions. The granular soils are often characterized by their cumulative particle mass as a function of particle size [45]. But due to the large number of parameters involved in soil behavior (particle shapes and their surface roughness, mechanical behavior of soil grains, ...), there is presently no general rule for the prediction of the mechanical properties of a soil from its particle size distribution. It is obvious that larger size spans lead to higher packing fraction by allowing for the pores of each size class to be partially filled by the particles of a lower-size class. The so-called Apollonian packings extend this hierarchical structure to an infinite number of classes composed of an increasing number of particles of decreasing size [4].

However, most effective properties of granular materials such as elastic moduli, shear stress, and compressibility crucially depend on the texture, in the sense of the contact network and force transmission, rather than packing fraction [46-49]. Recently, Voivret et al. investigated highly polydisperse packings of circular particles by means of contact dynamics simulations [50,51]. They found that, for all size spans, the highest packing fraction occurs for uniform particle volume distribution. They also showed that the number of rattlers, i.e., particles not involved in force transmission, increases with size span, and the force chains are mainly captured by large particles. For this reason, the shear strength of a noncohesive 
granular material is practically independent of particle size distribution. This clearly indicates that the mechanical properties are essentially controlled by the granular texture that needs therefore to be characterized as a function of both particle shape and size polydispersity.

In this paper, we are interested in the combined effects of particle shape and particle size distribution on the texture of isotropic granular packings by means of two-dimensional (2D) contact dynamics simulations. We consider pentagonal particles with varying degree of irregularity compared to regular pentagons. The particle size is given by the diameter of the circumscribed circle and the size polydispersity is defined from the size span for a uniform distribution of particle volume fractions. The samples are built by uniaxial compaction.

In order to reach the highest packing fraction, interparticle friction is set to zero. Frictionless packings have been extensively investigated as "reference systems" that highlight generic features of granular materials. In other words, they materialize the concept of random close packing (RCP) defined as the most compact and random packing. In the absence of friction, the particles are allowed to reach their lowest configurational energy without being trapped in metastable states as a result of frictional frustration. The RCP states have, however, only been investigated in sphere and disk packings. In this work we are concerned with RCP packings of polygonal particles. The microstructure is analyzed in terms of various statistical descriptors as a function of polydispersity parameters. Besides packing fractions and pair distribution functions, we will characterize in detail the distribution of sideside contacts and their aggregation into well-defied clusters encompassed by side-vertex contacts.

In the following, we present in Sec. II the numerical procedures, system characteristics, and preparation method. In Secs. III and IV we present our main findings on the space-filling and texture properties. We will conclude with a summary and perspectives of this work.

\section{NUMERICAL PROCEDURES}

\section{A. Numerical method}

The simulations were carried out by means of the contact dynamics (CD) method, which is suitable for simulating large assemblies of undeformable particles [52-55]. In this method, the rigid-body equations of motion are integrated by taking into account the kinematic constraints resulting from contact interactions. These interactions are characterized by three parameters: the coefficient of friction when the coefficient of friction is nonzero and the coefficients of normal and tangential restitution that control the rate of dissipation. An implicit timestepping scheme makes the method unconditionally stable. In contrast to the molecular dynamics method, in the CD method the possible overlaps between particles do not represent an elastic deflection, whose treatment then requires a fine time step to resolve correctly the overlap and thus ensure numerical stability. For this reason, the time step can be larger than that in the molecular dynamics method. In CD, an iterative algorithm based on nonlinear Gauss-Seidel iterations is used to determine the contact forces and particle velocities simultaneously at all potential contacts. The $\mathrm{CD}$ method has been extensively

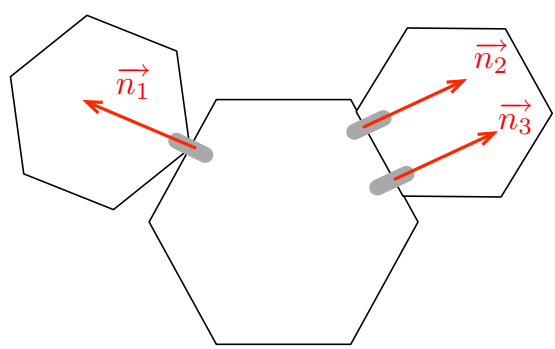

FIG. 1. (Color online) Two types of contact between two polygonal grains: (1) side-vertex contact (left) represented by a single contact point and a unit normal vector perpendicular to the side, and (2) side-side contact (right) represented by two contact points with their parallel unit normal vectors.

employed for the simulation of granular materials in two and three dimensions [18,19,56-65].

The $\mathrm{CD}$ method is based on implicit time integration of velocities but requires an explicit determination of the contact network at the beginning of each time step $[12,66]$. The contacts are defined from the small geometrical overlaps of the neighboring particles. The contact detection between two bodies consists of looking at the portions of space they occupy. The treatment of the mechanical interaction requires additionally the identification of a common tangent plane (a line in two dimensions). Of course, contact may take place through a larger contact zone than a single point. In $2 \mathrm{D}$ simulations of the present paper, the detection of contact between two convex polygonal bodies was implemented through the so-called shadow overlap method devised by Moreau, with reliability and robustness tested in several years of previous applications to various states of granular materials [67].

In detection of contacts between two polygons, three situations arise: (1) side-vertex contact, (2) side-side contact, and (3) vertex-vertex contact. A side-vertex contact is a "simple" contact as that between two disks. It corresponds to a single unilateral constraint between the two polygons, and the contact normal is perpendicular to the side; see Fig. 1. In contrast, a side-side contact is a "double" contact in the sense that it can be represented by two unilateral constraints. In other words, a double contact can be reduced to a couple of two simple contacts between the same polygons, and the normal direction is the normal to their common side, as shown in Fig. 1. In practice, this means that two forces are calculated at each side-side contact. But only their resultant and point of application are physically meaningful and independent of the positions of the two contact points. Finally, the vertex-vertex contacts are rare, but when they occur, they can be treated either as a simple contact by taking into account the path followed by the two particles or as a pair of double contacts, as discussed in more detail in [12].

\section{B. Packings}

\section{Shape and size parameters}

In our simulations, we use pentagons as reference particle shape. In fact, triangles and hexagons spontaneously assemble into crystalline structures. Squares tend to form columnlike 


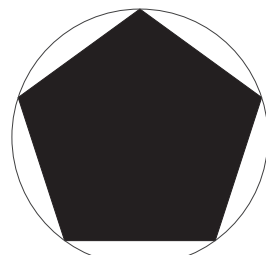

$\delta=0$

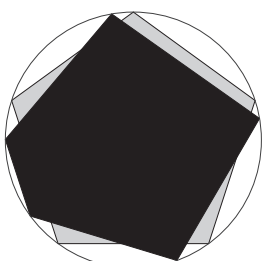

$\delta=0.5$

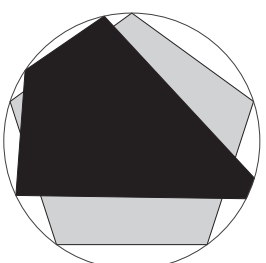

$\delta=1$
FIG. 2. Example of a regular pentagon $\delta=0$ transformed into irregular pentagons for two values of the parameter $\delta$ (see text).

structures, and polygons of seven and more sides are close to disks. In this sense, a pentagon may be considered as having the least roundedness without the pathological spacefilling properties of triangles, squares, and hexagons [12]. An irregular pentagon can be obtained by changing the angular positions of the vertices. Let $\theta_{0}$ be the position of the first vertex with respect to a reference frame. The angular position of a vertex $i$ is given by $\theta^{i}=\theta_{0}+2 \pi i / 5$. This regular pentagon can be transformed into an irregular pentagon by perturbing randomly the position of each vertex $i$ within an angular limit $\pm \delta \pi / 5$ :

$$
\theta^{i}=\theta_{0}+\frac{2 \pi}{5} i \pm \delta \frac{\pi}{5},
$$

where $\delta$ can be varied in the range $[0,1]$. Its value quantifies the degree of shape irregularity. Figure 2 shows two examples of irregular pentagons constructed from a regular one. In the following, we will refer to $\delta$ as "shape polydispersity" of the packing.

The size of a pentagonal particle is defined by the diameter $d$ of its circumscribed disk. It is varied in a range $\left[d_{\min }, d_{\max }\right]$ with a uniform distribution of particle volume fractions. Following [50], we define the size span $s$ of the distribution by

$$
s=\frac{d_{\max }-d_{\min }}{d_{\max }+d_{\min }} .
$$

The value $s=0$ corresponds to a monodisperse packing whereas $s=1$ corresponds to "infinite" polydispersity. In this paper, $s$ will be varied in the range $[0,0.9]$.

\section{Packing construction}

All packings are prepared according to the same protocol. For given values of $s$ and $\delta, 5000$ particles are generated with ten size classes with a uniform particle volume distribution. The number of particles in each class depends on $s$. We require that the following two representativity conditions be satisfied: (1) The number of particles in each class is above a minimum $N_{\min }$, and (2) The volume of each particle in a class $i$ is small compared to the total volume of the class. Note that these two conditions are equivalent for a quasimonodisperse distribution. For the most polydisperse packing ( $s=0.9$ ), we have 95 members in the class of largest particles, and 1350 members in the class of smallest particles. All classes have thus a high statistical representativity in terms of both the number and volume of particles, the total volume being the same in all size classes. As compared to $s=0.9$, the statistical representativity improves for smaller values of $s$ [50]. In each size class, the vertices of the pentagons on the circumscribed circle are randomly distributed within the range defined by $\delta$.

The particles are initially placed on a square network in a rectangular box of dimensions $l_{0} \times h_{0}$ and deposited under the action of the gravity $g$. Then, the gravity is set to 0 and the packings are subjected to vertical compression applied on
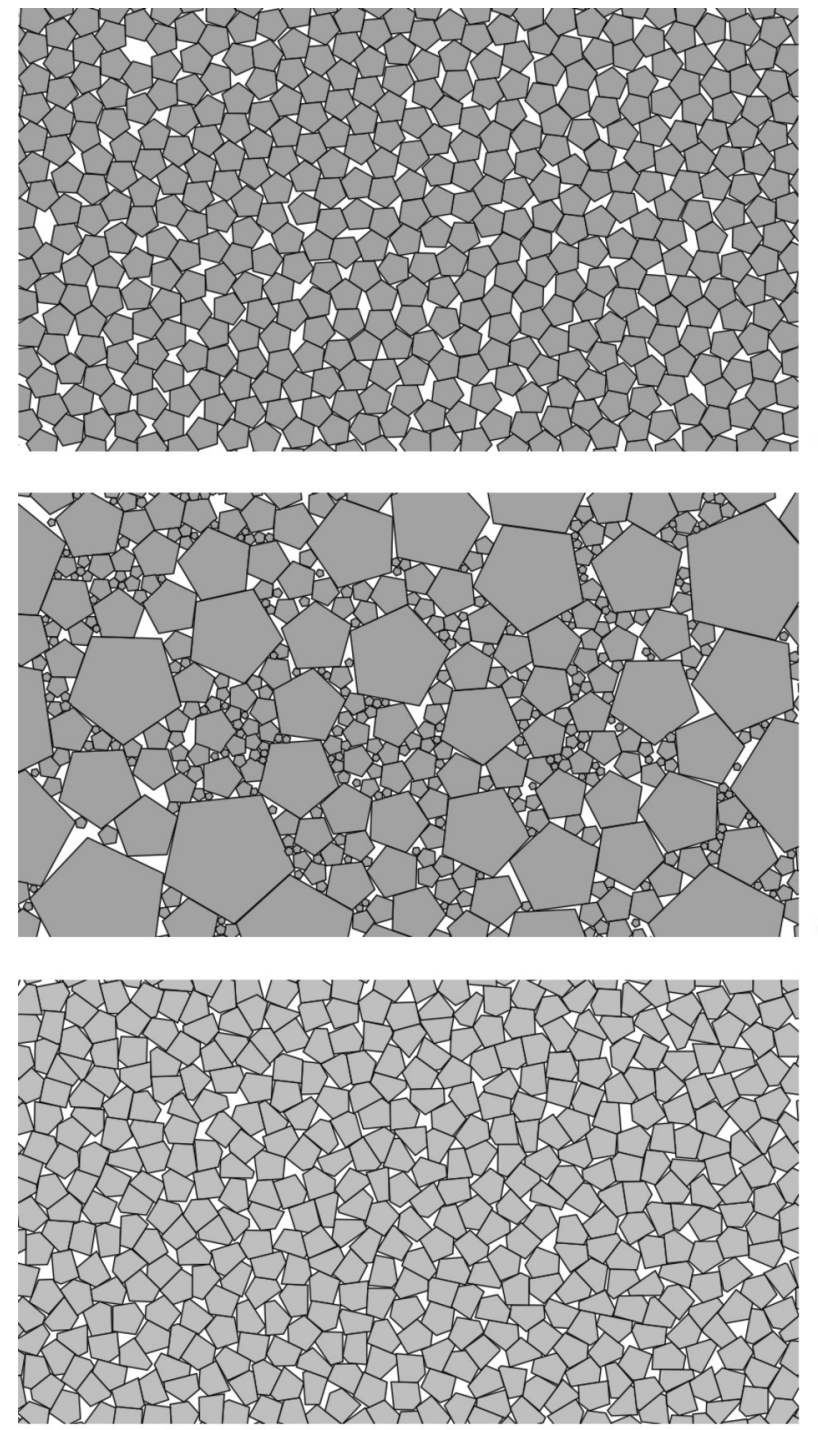

(c)

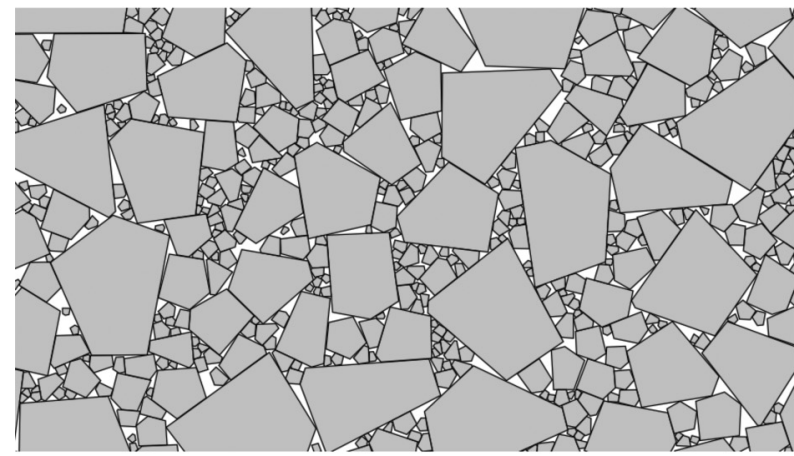

FIG. 3. Snapshots of dense packings at the end of compression: (a) $(s, \delta)=(0.01,0),($ b) $(s, \delta)=(0.9,0),(\mathrm{c})(s, \delta)=(0.01,1)$, and (d) $(s, \delta)=(0.9,1)$. 
the upper wall and where the left, bottom, and right walls are fixed. The friction coefficient between particles and with the walls is set to zero during the compression in order to obtain dense and nearly isotropic packings. The compression ends when a jammed mechanically stable configuration of particles is obtained.

The mean behavior for each set of parameters $s$ and $\delta$ is obtained by averaging over three independent data sets. 108 samples were prepared for six different values of the size span $s \in[0.01,0.9]$ and for six different values of shape polydispersity $\delta \in[0,1]$. Several snapshots of the packings obtained at the end of the compression are shown in Fig. 3 for $s=\{0.01,0.9\}$ and $\delta=\{0,1\}[68]$.

\section{SPACE-FILLING PROPERTIES}

In this section, we analyze the influence of size and shape polydispersity on various packing properties. We focus on the packing fraction $\rho_{m}=V_{p} / V$, where $V_{p}$ is the total particle volume and $V$ the box volume (area in two dimensions), radial pair distribution $g(r)$, and radial volume distribution $\rho(r)$ describing the correlations of packing fractions as a function of the radial distance $r$.

Figure 4 shows $\rho_{m}$ as a function of size span $s$ for all values of $\delta$. We observe that the packing fraction is an increasing function of both polydispersity parameters. The packing fraction increases from 0.85 to 0.89 as the size span is increased for $\delta=0$ and from 0.87 to 0.90 for $\delta=1$. Conversely, for $\delta$ from 0 to 1 , the packing fraction varies from 0.85 to 0.87 for $s=0.01$ and from 0.89 to 0.90 for $s=0.9$. Hence, the effect of shape prevails at low size polydispersity $(s<0.4)$ whereas it becomes less effective as size polydispersity increases. Furthermore, higher values of the packing fraction are reached with increasing size span rather than increasing shape polydispersity.

While the increase of $\rho_{m}$ with $s$ is simply related to partial filling of pore space between large particles by smaller particles, its increase with $\delta$ is much less evident. This behavior may be described in terms of the "angularity" of the particles. Random-shaped pentagons have at least one internal angle well below the average angle $\pi / 5$, thus allowing the corresponding vertex to intrude easily into free volumes between particles. This is also the reason why a disordered monodisperse packing

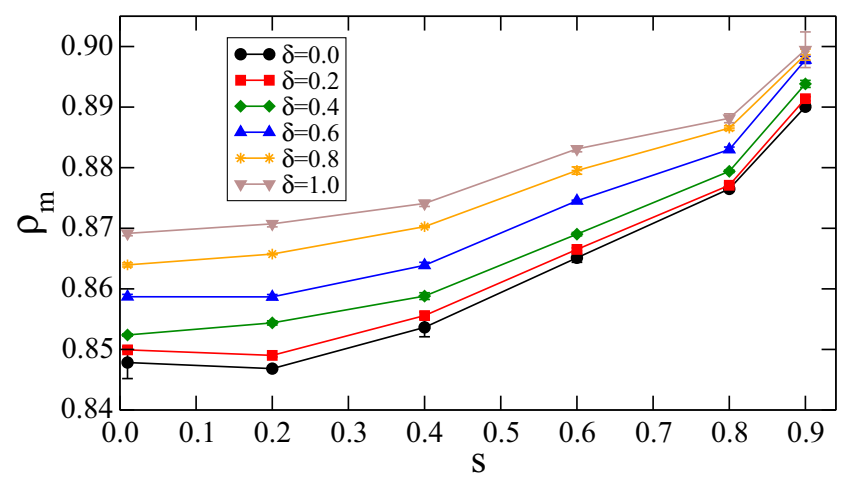

FIG. 4. (Color online) Packing fraction $\rho_{m}$ as a function of size span $s$ for all values of shape polydispersity $\delta$.

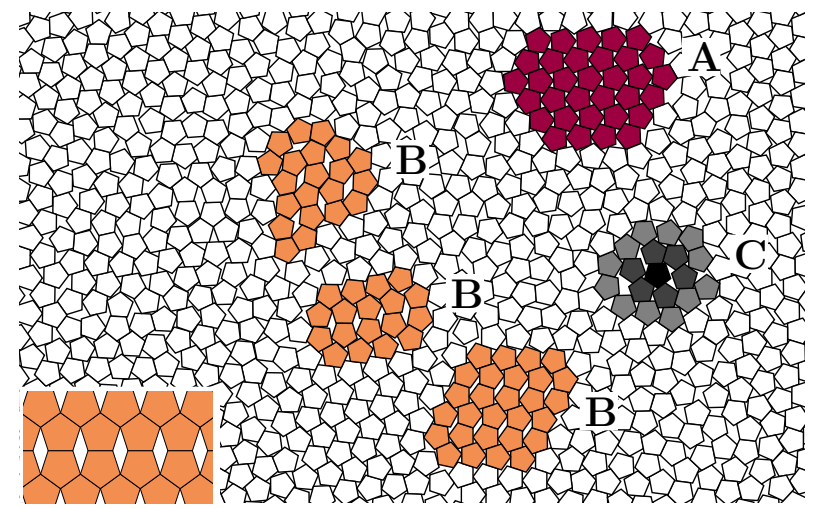

FIG. 5. (Color online) Examples of crystalline or quasicrystalline cores in a nearly monodisperse packing of regular pentagons: (A) (red) densest configuration (packing fraction $\simeq 92$ ) involving both positional and orientational order of the pentagons; (B) (orange) "slipped" or slightly randomized examples of the fully ordered configuration shown in the bottom-left inset with a packing fraction $\simeq 0.85$; (C) (gray) typical local environment of the pentagons characterized by a local triangular positional symmetry and random orientations of neighboring particles.

of frictionless disks has a packing fraction $(\simeq 0.82)$ below that of regular pentagonal particles (0.85) [50]. In other words, sharper corners play the same role as the points of large curvature on a particle, used in [32] to explain the increase of packing fraction as a function of distortion of particle shapes from a perfectly circular or spherical shape.

A dense disordered packing of monodisperse regular pentagons as the one shown in Fig. 3(a) may be described as a random close packing of pentagons. Various crystalline packings of monodisperse pentagons can be constructed geometrically but many of such packings are singular or mechanically unstable [69]. However, Fig. 3(a) reveals several quasicrystalline domains with local packing fractions above or equal to the average packing fraction $\rho_{m} \simeq 0.85$. Two such crystalline phases are highlighted in Fig. 5. The first phase has a packing fraction $\simeq 0.92$ and was described in Ref. [69] as the densest crystalline phase of $2 \mathrm{D}$ pentagon packings. Its high packing fraction is slightly above that of triangular disk packings $(\simeq 0.91)$ mainly due to the intruding-corner effect described previously. The second crystalline phase is a "slipped" variant of a geometrically constructed crystalline phase (inset to Fig. 5). The packing fraction of the nonslipped phase is 0.854 . In both phases, not only the centers of the pentagons but also their orientations display long-range order.

Apart from the above two crystalline phases, the most common structure observed in Fig. 5 is characterized by a triangular symmetry as in regular monodisperse packings of hexagons, but without the orientational order of neighboring pentagons. This structure was observed in experiments [70] and it may be obtained from the densest crystalline phase by randomly rotating the pentagons. Although the crystalline cores tend to increase the mean packing fraction, the mismatch between the fivefold symmetry of the particles and the sixfold symmetry of the packing may be considered as the main origin of disorder and thus the reduction of the packing fraction of pentagonal particles from 0.92 to 0.85 . 


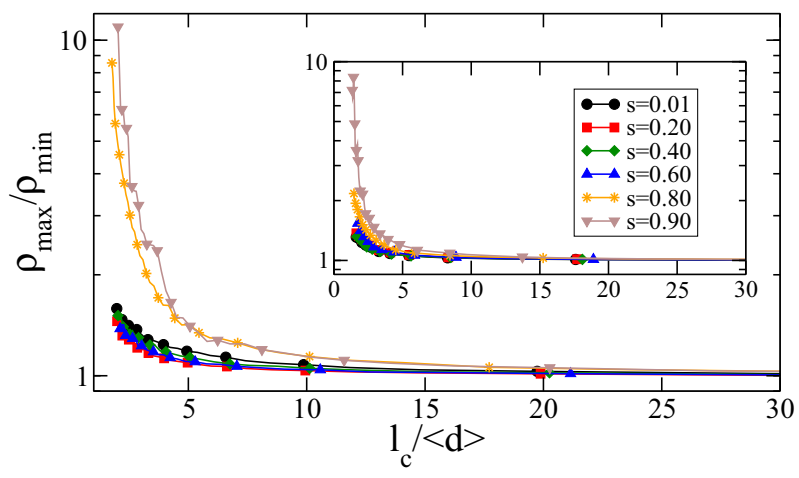

FIG. 6. (Color online) Variation of the ratio $\rho_{\max } / \rho_{\min }$ as a function of the normalized grid spacing for $\delta=0$ and $\delta=1$ (inset).

Visual inspection of Figs. 3(a) and 3(c) suggests also that the packing of irregular pentagons is more homogeneous than the packing of regular pentagons. The shape symmetry in the latter case together with steric exclusions enhances the arching effect and leads to large pores. The relative homogeneity of the packing of irregular pentagons can thus be described by stating that the steric hindering constraints are generally less effective in this case. Many methods have been developed to describe quantitatively the homogeneity of a disordered microstructure. In the following, we employ the density fluctuation method introduced and used by Saint-Cyr et al. [71]. In this method, the packings are subdivided into $n_{c}^{2}$ cells using a square grid, where $n_{c}$ is the number of cells along each direction of linear size $l_{c}$ varied from $l_{c}=2 d$ to $l_{c}=l_{0}=60 d$. For this subdivision, we compute $\rho_{\min }$ and $\rho_{\max }$ defined as, respectively, the minimum and maximum values of the packing fraction evaluated for all cells. The ratio $\rho_{\max } / \rho_{\min }$ is a measure of structural inhomogeneity as a function of $l_{c}$.

Figure 6 shows $\rho_{\max } / \rho_{\min }$ as a function of the normalized cell size $l_{c} /\langle d\rangle$ for all values of $s$ at $\delta=0$ and $\delta=1$. We see that, for all values of $\delta$ and $s, \rho_{\max } / \rho_{\min }$ declines towards a constant plateau value $\simeq 1$ as $l_{c}$ is increased. This behavior reflects the decrease of $\rho_{\max }$ and increase of $\rho_{\text {min }}$ with $l_{c}$. For all values of $s$ and $\delta$, two regimes can clearly be distinguished: (1) A rapid decrease of $\rho_{\max } / \rho_{\min }$ up to $l_{c} \simeq 5\langle d\rangle$ and (2) a slow decrease towards 1 . These two scales seem to correspond, respectively, to (1) the particle environments and (2) the mesoscopic scales due to crystalline domains and their spatial extensions, as well as the effects related to particle orientations and side-side contacts. The inhomogeneity is higher for packings of irregular and more polydisperse pentagons. For example, for $l_{c}=2\langle d\rangle$, we have $\rho_{\max } / \rho_{\min }<2$ for $s<0.8$ whereas $\rho_{\max } / \rho_{\min } \simeq 10$ at large values of $s$.

\section{Radial distribution functions}

The effect of shape and size polydispersity on the local ordering of particle positions can be evaluated by means of the radial distribution function $g(r)$ of the radial positions $r$ of particle centers:

$$
g(r)=\frac{n(r)}{n},
$$
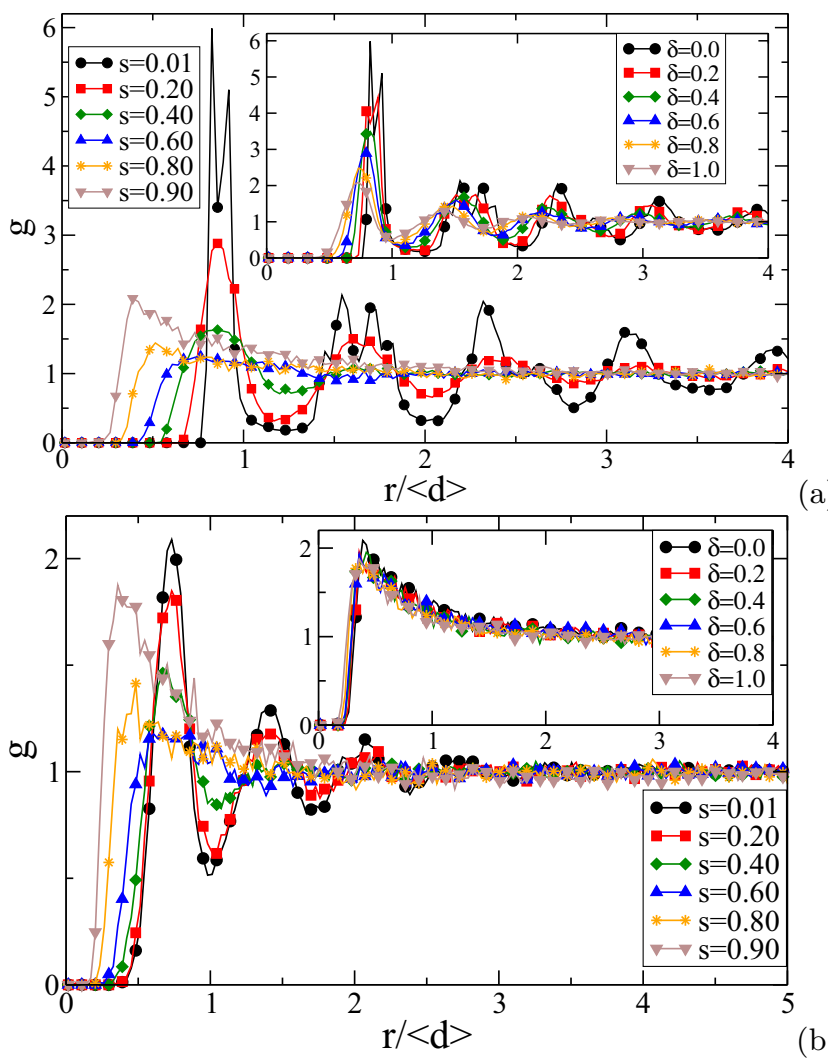

FIG. 7. (Color online) Radial distributions $g$ as a function of radial distance $r$ normalized by the average particle diameter $\langle d\rangle$ for $\delta=0$ (a) and $\delta=1$ (b) for all values of size span $s$. The inset of (a) shows $g(r)$ for $s=0.01$ at all values of $\delta$. The inset of (b) shows $g(r)$ at $s=0.9$ for all values of $\delta$.

where $n(r)$ is the average number density of particle centers at a distance $r$ from a particle and $n$ is the average number density of particles. The variations of $g(r)$ as a function of $r$ reflect thus the average placement of the surrounding particles.

The function $g(r)$ for $\delta=0$ and $\delta=1$ is shown in Figs. 7(a) and 7(b), respectively, for all values of $s$. The insets of Figs. 7(a) and 7(b) show also $g(r)$ for $s=0.01$ and $s=0.9$ for all values of $\delta$. Note that the radial distances have been normalized by the mean diameter $\langle d\rangle$. For $s<0.4$ we observe the signature of short-range order with regular peaks of decreasing amplitude. In this range, the position of the first peak is nearly independent of the value of $s$, and the amplitude decreases as $\delta$ increases. We also note that in this range of the values of $s$, the peaks shift to smaller distances with increasing $\delta$. This behavior is consistent with more interlocking of the particles and larger "intrusion" of increasingly sharper corners of the particles, as discussed previously. For $s=0.01$ (quasimonodisperse packings) and for $\delta=0$ or 0.1 , the first peak splits into two narrow subpeaks of nearly the same amplitude. These subpeaks occur at $r /\langle d\rangle=\cos \pi / 5 \sim 0.8$ and $r / d \propto 0.5(1+\cos \pi / 5) \sim 0.9$, which are the intercenter distances between pentagons for side-side and side-vertex contacts, respectively. In a similar vein, the second peak is split into four subpeaks. For $s>0.4$, only one peak survives and, due to polydispersity, it shifts to a distance below $\langle d\rangle$ with an increasing amplitude as a function of $s$. 
This increase of the amplitude of $g$ with high polydispersity may be attributed to the uniform distribution of particle volumes. Indeed, as the polydispersity increases, the number of particles in the small-size classes grows rapidly and leads to increasing local ordering of small particles assembled in the pores between larger particles. Hence, the size span $s=0.4$ is the point of transition from a polydispersity where a single size generation governs the packing to a polydispersity with two and then three size generations. The members of the second generation are those fitting into the pores of particles from the first generation. It is also worth noting that, at high polydispersity as observed in Fig. 8, the radial distributions are quite similar for all values of $\delta$. This means that, as for packing fraction, the shape polydispersity plays a minor role at high polydispersity.

As far as the space-filling aspect in highly polydisperse media is concerned, it is also convenient to consider the packing fraction as a function of radial positions $r$ of the particles. The radial volume fraction distribution $\rho(r)$ can be calculated inside circular shells of increasing radius $r$ centered on particle centers. Figure 8 shows $\rho(r) / \rho_{m}$ for $\delta=0$ and $\delta=1$ for all values of size span $s$. The insets show also $\rho(r) / \rho_{m}$ at $s=0.01$ and $s=0.9$ for all values of $\delta$. The plateau at low $r$ corresponds to the interior of the particles where $\rho(r)=1>\rho_{m}$. The volume fraction distribution oscillates between peaks and valleys of decreasing amplitude. The valleys represent the void space between a particle and its first or higher-order neighboring particles. We see that $\rho(r)$
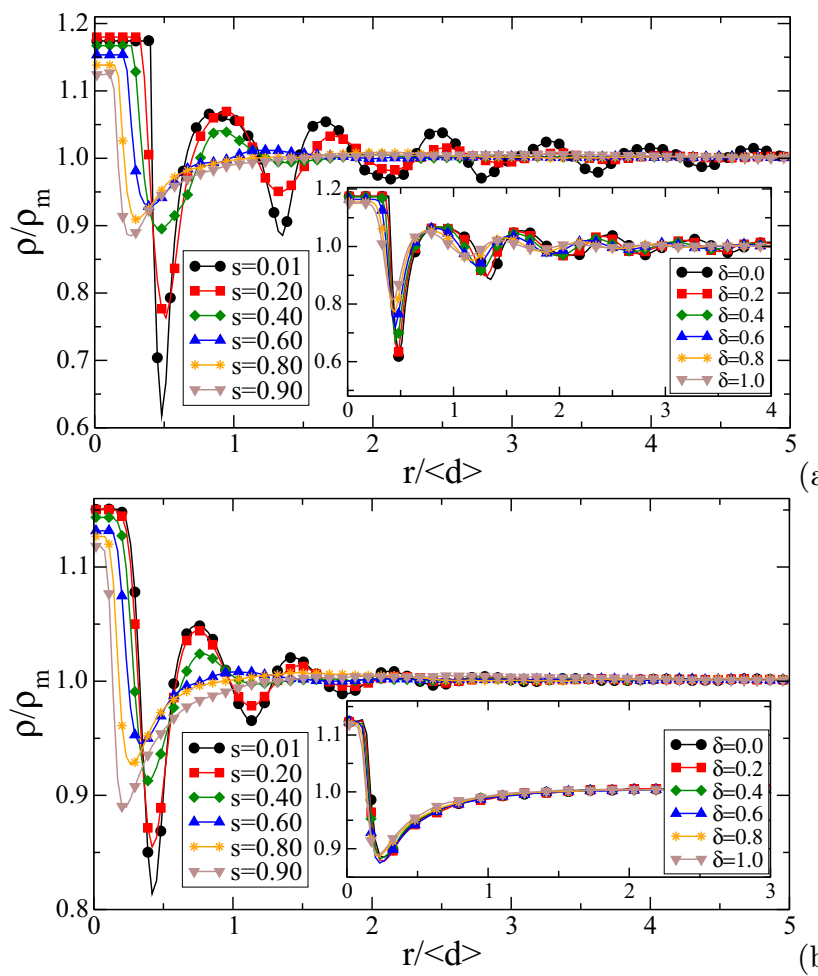

(a)

FIG. 8. (Color online) Radial volume fraction distribution $\rho$ normalized by the average packing fraction as a function of radial distance $r$ for $\delta=0$ (a) and for $\delta=1$ (b) for all values of size span $s$. The inset of (a) shows $\rho$ at $s=0.01$ for all values of $\delta$. The inset of (b) shows $\rho$ at $s=0.9$ for all values of $\delta$. tends to the mean packing fraction $\rho_{m}$ as $r$ increases. But, the convergence to $\rho_{m}$ is faster and thus the correlation length becomes smaller as both $\delta$ and $s$ increase. At high size polydispersity, we mainly observe a marked valley following the plateau and a smooth increase towards $\rho_{m}$.

\section{CONTACT NETWORKS AND FORCE TRANSMISSION}

The contact network can be described in terms of various statistical descriptors pertaining to the force-bearing network of particles such as the coordination number $Z$ (i.e., mean number of contact neighbors per particle), the proportion $P_{c}$ of particles with exactly $c$ contact neighbors, and force distributions [72]. We consider in this section these geometrical and mechanical descriptors in order to identify the signature of particle shape and size polydispersity.

\section{A. Connectivity}

Figure 9 shows a map of the particle coordination numbers for $(s=0.01, \delta=0)$ and $(s=0.9, \delta=1)$. Note that only the "contact neighbors," i.e., the number of neighbors having a side-vertex or a side-side force-bearing contact with each particle, are counted. The contact network topology is very inhomogeneous in both monodisperse and polydisperse packings. The proportion of particles having $c$ contact neighbors is plotted in Fig. 10. We have $\sum_{c} P_{c}=1$ and $\sum_{c} c P_{c}=Z$. We see that $P_{c}$ is nearly independent of $\delta$ for all values of $s$. $P_{3}$ increases with $s$ from 0.1 to 0.5 whereas both $P_{4}$ and $P_{5}$ decline from 0.4 to 0.2 and 0.1 , respectively. $P_{7}$ and $P_{8}$ increase with $s$ but remain negligibly small with values as small as 0.05 . In the same way, $P_{2} \simeq 0$ and for this reason it was not shown in Fig. 10. The slower decrease of $P_{4}$ compared to $P_{5}$ as a function of $s$ indicates that the population of particles
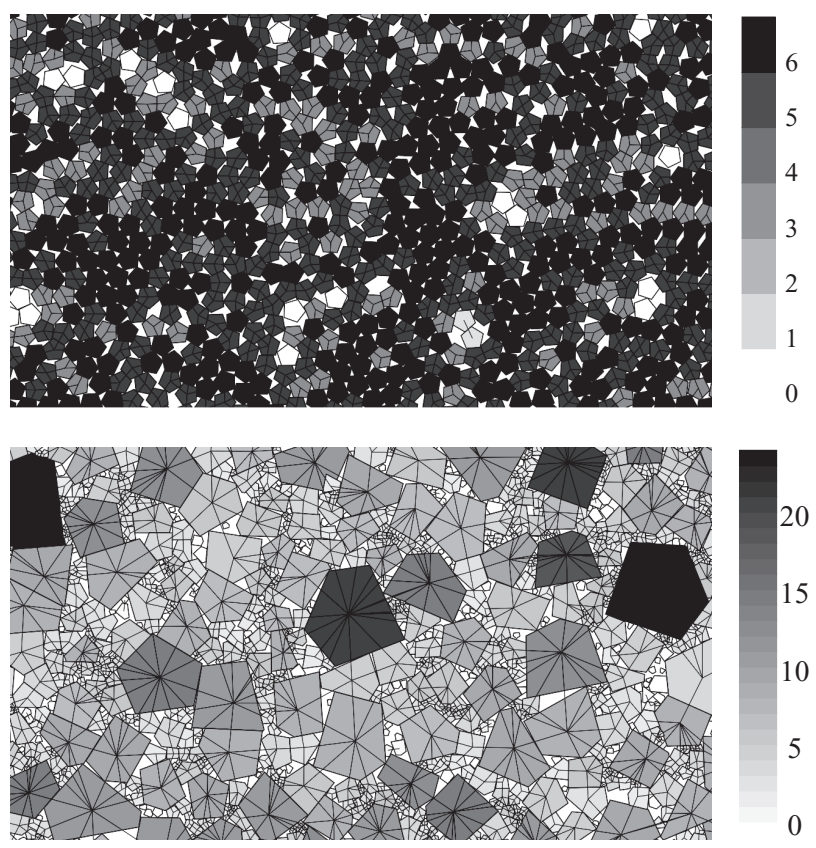

FIG. 9. Grey level map of particle connectivities and its scale bar for $(s=0.01, \delta=0)$ (a) and $(s=0.9, \delta=1)$ (b). Floating particles are in white and the grey level is proportional to coordination number. 


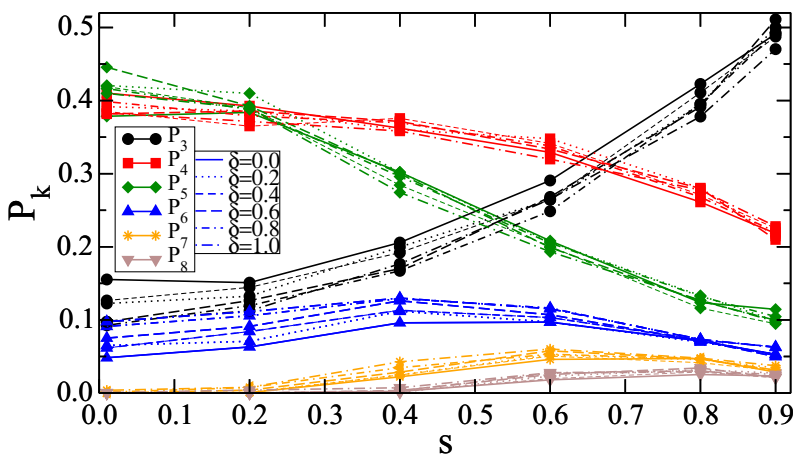

FIG. 10. (Color online) Proportion $P_{c}$ of particles having $c$ contact neighbors as a function of $s$ for all values of $\delta$.

with four neighbors gains new members from the population of coordination number 5 but loses even more members to the population of coordination number 3 . Hence, as the size polydispersity becomes higher, an increasing number of particles are equilibrated by three forces, approximately one-third of them being side-side contacts, as we will see below.

Figure 11 shows $Z$ as a function of $s$ for all values of $\delta$. For all values of $\delta, Z$ is nearly constant for $s<0.4$, and it slightly declines for $s>0.4$. In contrast, for each given value of $s, Z$ increases with $\delta$. This observation is consistent with a higher interlocking of particles at higher shape polydispersity.

The nearly constant value of $Z$ despite increasing size polydispersity is surprising. It may also seem surprising that its value is well below 6 , which is the expected coordination number of a system of noncircular frictionless particles (with three degrees of freedom) [73]. A similar behavior was observed for ellipses, ellipsoids [16], and tetrahedra [74], which were found to have an average coordination number below their respective isostatic values. This apparent departure of ellipsoids from isostatic behavior was attributed to the presence of floppy modes, which provide vanishing restoring force, whereas for tetrahedra it was attributed to the varying degrees of rotational constraint by discrete contact topologies.

We would like to introduce here a different interpretation. The point is that the side-side contacts do not have the same status as side-vertex contacts. The normal force at a side-vertex contact has a single point of application that coincides with the

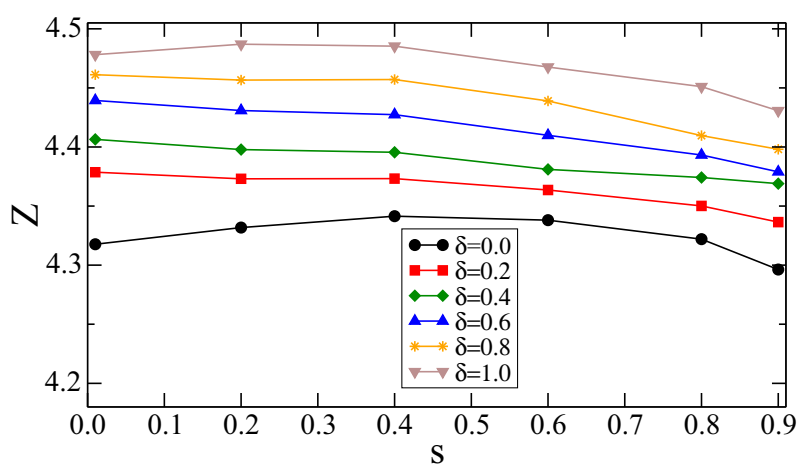

FIG. 11. (Color online) Coordination number $Z$ as a function of $s$ for all values of $\delta$.

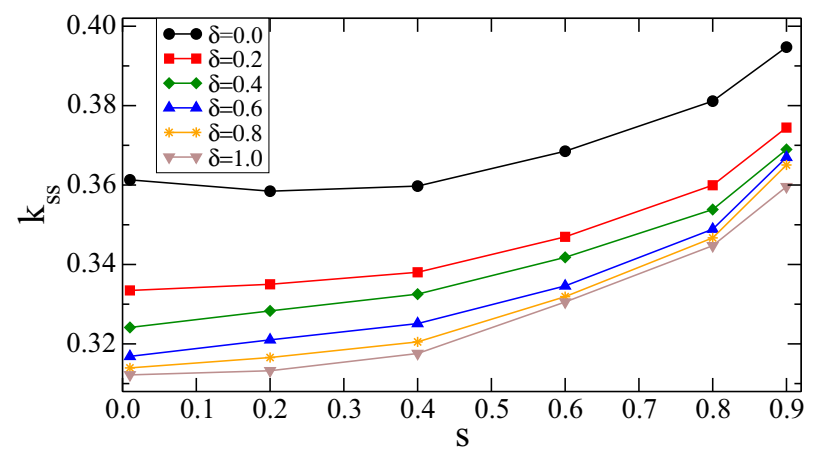

FIG. 12. (Color online) Proportion $k_{s s}$ of side-side contacts as a function of $s$ for all values of $\delta$.

contact point. In contrast, at a side-side contact, the point of application of the normal force is unknown and is determined by the balance of forces. In other words, a side-side contact represents two geometrical constraints and, in this respect, it should be counted twice when one enumerates the number of unknowns. Let $k_{s s}$ be the proportion of side-side contacts, $N_{f}$ the number of degrees of freedom per particle, and $Z$ the number of contact neighbors. On average, $k_{s s} Z / 2$ contacts per particle are side-side whereas $\left(1-k_{s s}\right) Z / 2$ contacts are sidevertex. Hence, the total number of constraints per particle is $k_{s s} Z+\left(1-k_{s s}\right) Z / 2=\left(1+k_{s s}\right) Z / 2$. In an isostatic system, the number of constraints is equal to the number of degrees of freedom. As a result, we have

$$
Z=\frac{2 N_{f}}{1+k_{s s}}
$$

This relation correctly yields $Z=6$ for $k_{s s}=0$, but its value is below 6 when side-side contacts occur. Figure 12 displays the proportion of side-side contacts as a function of $s$ for all values of $\delta$. The approximate value of $k_{s s}$ is $1 / 3$. But we see that it is smaller at higher $\delta$ and slightly increases with $s$. By virtue of Eq. (4), this evolution of $k_{s s}$ is consistent with the observed behavior of $Z$ as a function of $s$ in Fig. 11 .

Equivalently, we may introduce a "connectivity number" $Z_{c}$ defined as the number of constraints per particle by counting the side-vertex contacts once and side-side contacts twice. We get

$$
Z_{c}=\left(1+k_{s s}\right) Z \text {. }
$$

According to Eq. (4), in an isostatic system we thus get $Z_{c}=$ $2 N_{f}$. This connectivity number in our system is $Z_{c} \simeq 6$.

The correlation between particle size and connectivity can be obtained by considering the average number $Z\left(d_{r}\right)$ of contact neighbors in the class of particle size within the interval $\left[d_{r}-\Delta d_{r} / 2, d_{r}+\Delta d_{r} / 2\right]$, where $d_{r}=$ $\left(d-d_{\min }\right) /\left(d_{\max }-d_{\min }\right)$ is the mean reduced size of the particles in each size class. Figure 13 shows $Z\left(d_{r}\right)$ for all values of $s$ with $\delta=0$. At low values of $s=0.01$, the polydispersity is negligible and all size classes have practically the same coordination number $(\simeq 4.2)$. At larger values of $s, Z$ increases almost linearly with $d_{r}$. The particles smaller than $d_{r}=0.2$ have less neighbors than $\sim 4.2$ whereas the mean number of neighbors for larger particles increases to nearly 13 for $s=0.9$. 


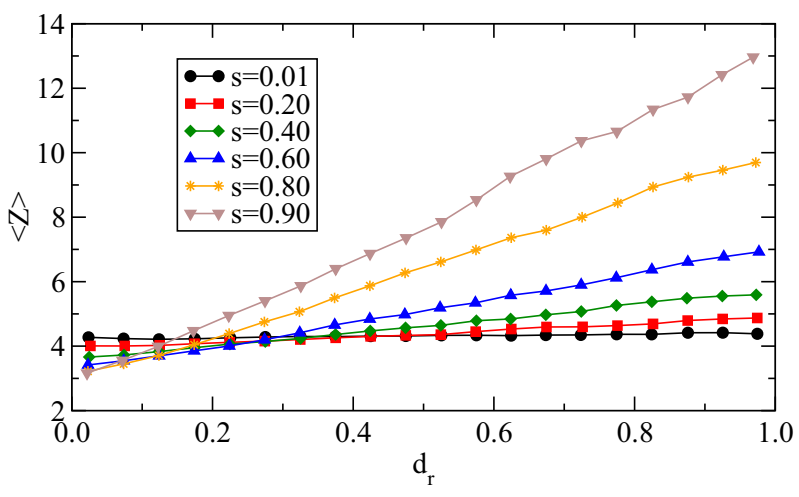

FIG. 13. (Color online) Linear correlation between the coordination number $Z$ and reduced particle size $d_{r}$.

In the analysis of the connectivity and coordination only the force-bearing contacts are considered. The purely "geometrical contacts," where the normal force is strictly zero, and the "floating particles," that have no force-bearing contacts, are thus excluded from the statistics. The proportion $P_{f}$ of floating particles is, however, an interesting fabric property as it provides an indication of the degree of arching in a granular material. In polydisperse materials, the arching effect is enhanced by size dispersion and we thus expect an increasing number of particles to be excluded from the force network. Figure 14 shows $P_{f}$ as a function of $s$ in our packings for all values of $\delta$. We see that $P_{f}$ is quite small for $s<0.4$ but increases up to nearly 0.25 for $s$ varying from 0.4 to 0.9 . It is also remarkable that $P_{f}$ is practically independent of $\delta$. Note that most floating particles are small particles representing a small volume fraction of the packing, as can be observed in Fig. 9(b).

\section{B. Clustering}

The role of side-side contacts was briefly analyzed above in connection with the connectivity and crystallized cores. Since nearly one-third of contacts are of side-side type, the issue that we would like to address in this section is how those contacts are organized within our samples of frictionless pentagons. Do the side-side contacts percolate throughout the packing? How do the size and shape polydispersity affect their spatial arrangement? We thus consider the particles bonded by

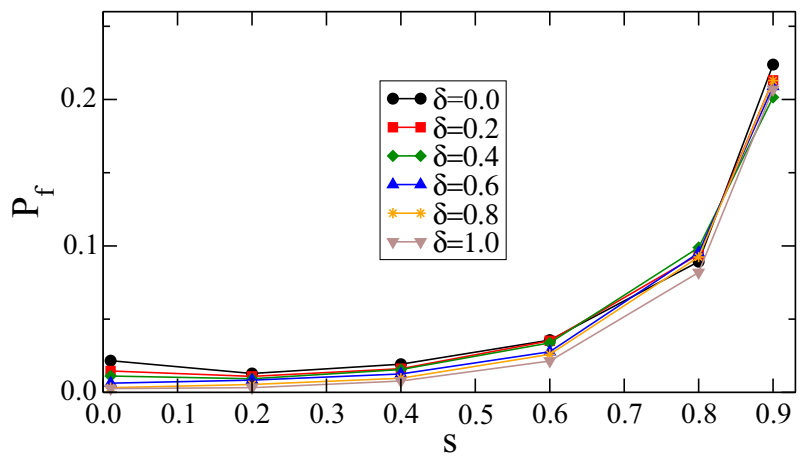

FIG. 14. (Color online) Proportion $P_{f}$ of floating particles as a function of $s$ for all values of $\delta$. side-side contacts and identify clusters of particles in which each particle is bonded by at least one side-side contact to another particle of the same cluster. Two such clusters are disjoint if they have no common side-side contact, i.e., when the boundary particles of one cluster are connected only by side-vertex contacts with the other cluster.

Four examples of the clusters are shown in Fig. 15 for four pairs of the values of $s$ and $\delta$. The side-side contacts are marked by a line joining the centers of the partner particles. In exception to a few number of particles having no side-side contact, most particles belong to a cluster. No cluster encompasses the whole packing, showing that the side-side contacts do not percolate. At low size and shape polydispersity, two types of clusters can be distinguished: (1) crystalline domains, as those identified in Fig. 5, and (2) long zigzag chains. At low size polydispersity and high shape polydispersity, the clusters are small. But at high size polydispersity we observe well-formed large clusters.

Figure 16 shows the number $N_{c}$ of clusters as a function of $s$ for all values of $\delta$. We see that, irrespective of $\delta, N_{c}$ remains constant as long as $s<0.4$ and it declines quickly to a much lower number as $s$ increases. We also see that, for a given value of $s, N_{c}$ increases with $\delta$. At the same time, as expected and shown in the inset of Fig. 16, the mean number of particles $\left\langle N_{p}\right\rangle_{c}$ per cluster increases with $s$. Since the shapes and sizes of the clusters seem to vary with polydispersity, it is interesting to evaluate their longest dimension $\ell_{\max }$ and shortest dimension $\ell_{\min }$. Figure 17(a) shows $\ell_{\max }$ and $\ell_{\min }$ normalized by the maximum particle diameter $d_{\max }$ as a function of $s$ for all values of $\delta$. We see that, due to disorder, both $\ell_{\max }$ and $\ell_{\min }$ decline as $s$ and $\delta$ increase. $\ell_{\max }$ is 13 particle diameters for the most regular packings (i.e., for $s=0.01$ and $\delta=0$ ) and only 5 diameters for the least regular ones (i.e., for $s=0.9$ and $\delta=1$ ). We also remark that the shape polydispersity affects the mean size of the clusters at low size polydispersity. As in the case of packing fraction, the shape polydispersity becomes less influent as the size polydispersity increases. Indeed, for nearly monodisperse packings $\ell_{\max } / d_{\max }$ declines from 13 to 7 as $\delta$ varies from 0 to 1 whereas it declines from 5 to 3 for $s=0.9$.

The mean sphericity of the clusters can also be evaluated by averaging their aspect ratios $a=\ell_{\max } / \ell_{\min }$. The mean aspect ratio $\langle a\rangle$ of the clusters is plotted as function of $s$ for all values of $\delta$ in Fig. 17(b). $\langle a\rangle$ declines with both $s$ and $\delta$, thus showing that the clusters become less anisotropic as structural disorder increases. The clusters have, however, random orientations. Figure 17(b) clearly shows that the shape anisotropy of the clusters even at high polydispersity is a consequence of their columnlike ordering.

\section{Force transmission}

From the force-balance viewpoint, it may be argued that if the side-side contacts tend to capture the strong force chains, then the anisotropy of the clusters is simply a reflection of force correlations. This point is checked in Fig. 18(a) where we have plotted the mean forces $\left\langle f_{s s}\right\rangle$ and $\left\langle f_{s v}\right\rangle$ at the side-side and side-vertex contacts, respectively, as a function of $s$ for all values of $\delta$. It is seen that both average forces are nearly independent of $\delta$, and although the proportion 

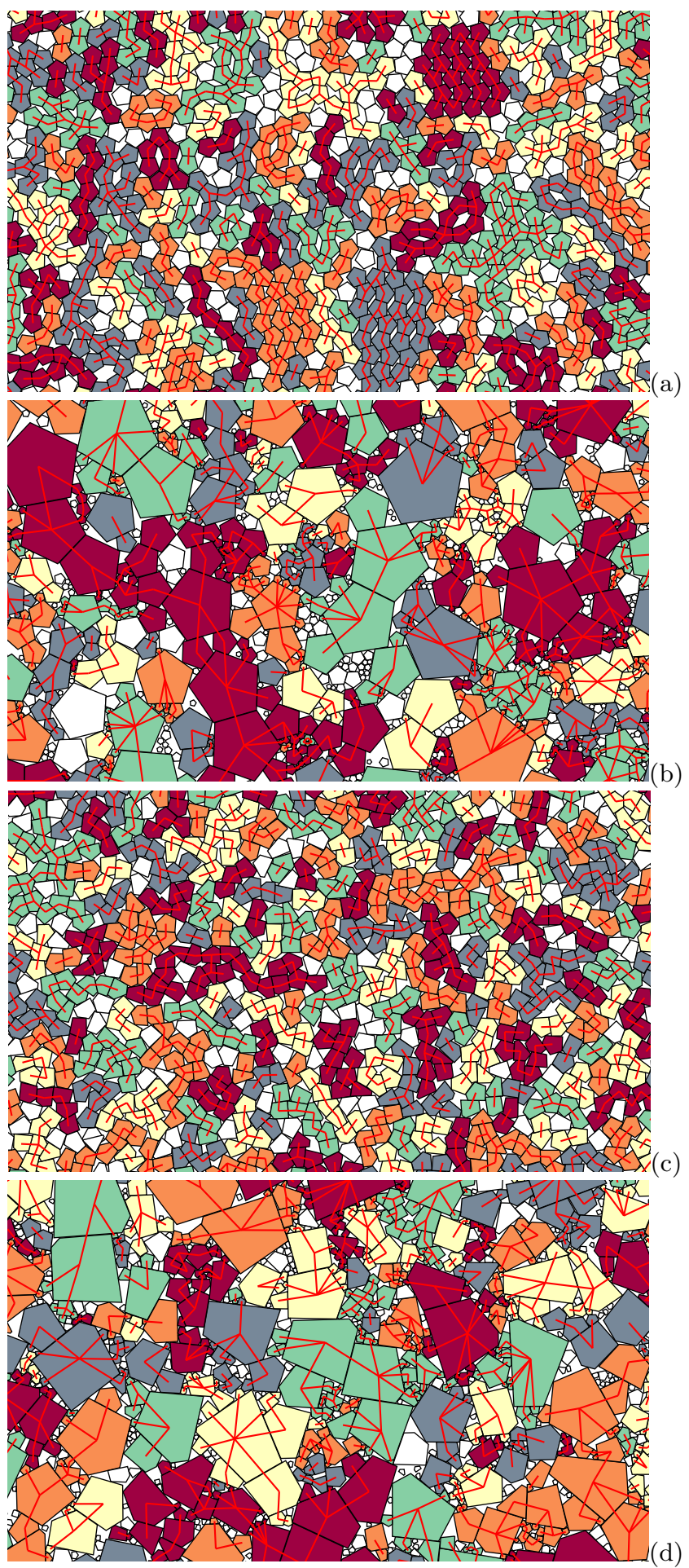

FIG. 15. (Color online) Clusters of particles bonded by at least one side-side contact for $(s, \delta)=(0.01,0)(\mathrm{a}),(s, \delta)=(0.9,0)$ (b), $(s, \delta)=(0.01,1)(\mathrm{c})$, and $(s, \delta)=(0.9,1)(\mathrm{d})$. Disjoint clusters are represented in different colors (green, orange, light slate gray, and cardinal red). The side-side contacts are marked by a thick black line joining the centers of their partner particles. The floating particles are in white.

of side-side contacts is almost half of that of side-vertex contacts, $\left\langle f_{s s}\right\rangle$ is larger than $\left\langle f_{s v}\right\rangle$. Hence, it is plausible to

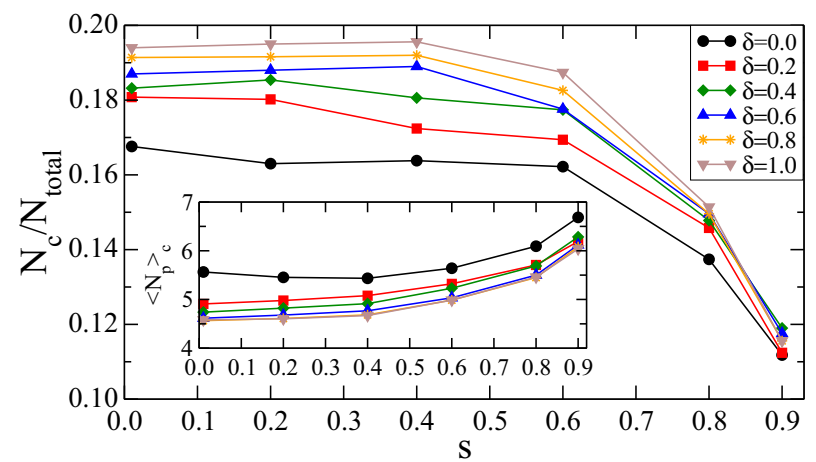

FIG. 16. (Color online) Number $N_{c}$ of clusters as a function of $s$ for all values of $\delta$. The inset shows the mean number $\left\langle N_{p}\right\rangle_{c}$ of particles per cluster as a function of $s$.

attribute the anisotropy of the clusters of side-side contacts to the chainlike structure of strong forces. The difference $\left\langle f_{s s}\right\rangle-\left\langle f_{s v}\right\rangle$ declines as $s$ increases, in agreement with the decreasing anisotropy of the clusters when size polydispersity increases.

Figure 18(b) shows $\left\langle f_{s s}\right\rangle /\langle f\rangle$ and $\left\langle f_{s v}\right\rangle /\langle f\rangle$ as a function of $s$. We see that the normalized forces have a nearly constant value (with a slight decrease of $\left\langle f_{s s}\right\rangle /\langle f\rangle$ as $s$ increases), indicating that, despite gradual change in polydispersity, almost the same fractions of the mean force are sustained by the side-side and side-vertex contacts. We also note that $\left\langle f_{s s}\right\rangle$ is higher than $\left\langle f_{s v}\right\rangle$ but remains always below $2\left\langle f_{s v}\right\rangle$. Since side-side contacts are represented as
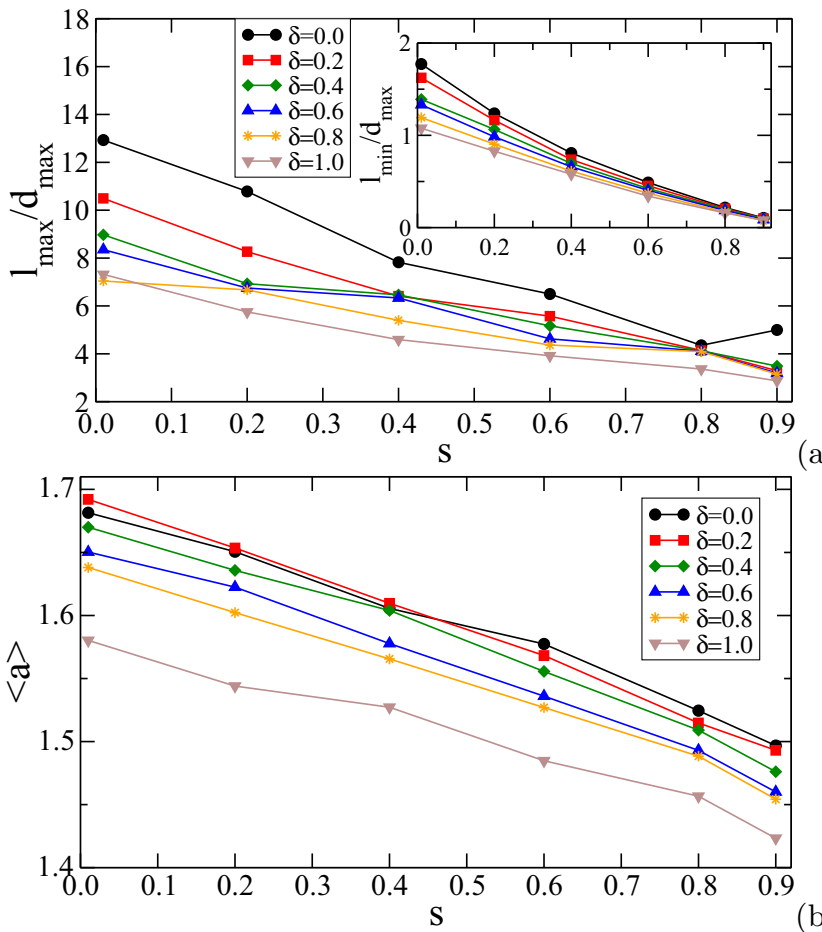

(a)

FIG. 17. (Color online) (a) Average values of the longest and shortest dimensions of the clusters normalized by the largest particle diameter $d_{\max }$ (a) and their mean aspect ratio $\langle a\rangle$ (b) as a function of $s$ for all values of $\delta$. 

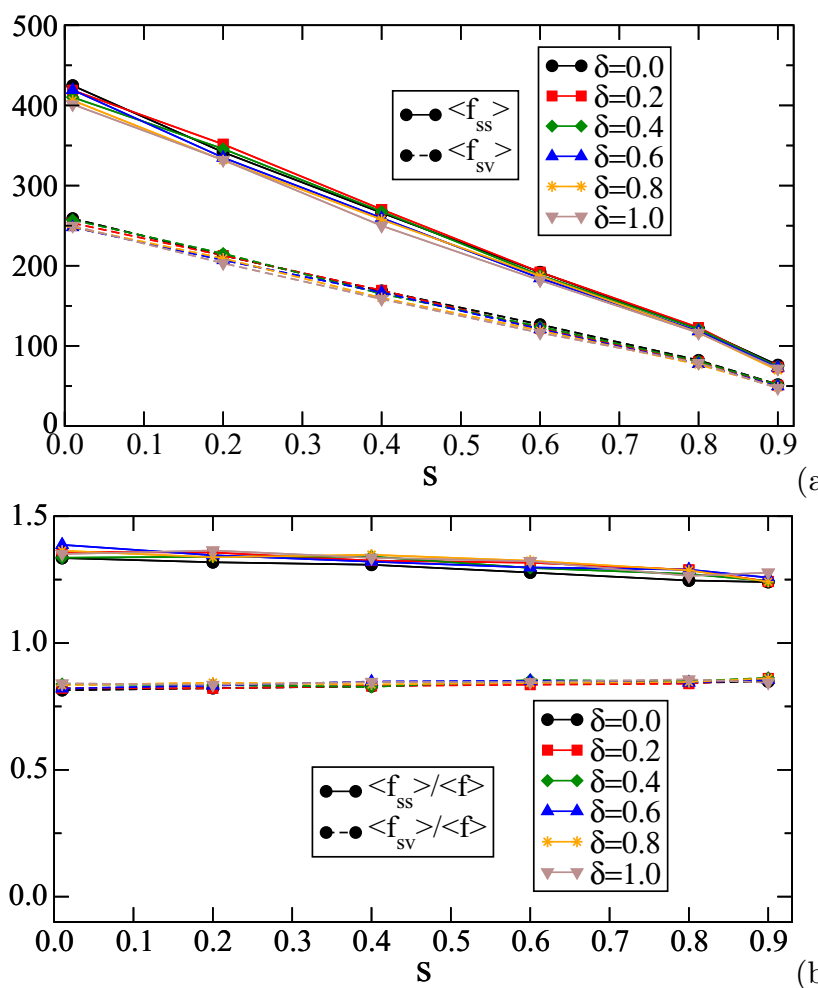

(a)

FIG. 18. (Color online) (a) Average side-side force $\left\langle f_{s s}\right\rangle$ and side-vertex force $\left\langle f_{s v}\right\rangle$ as a function of $s$ for all values of $\delta$. (b) The same data normalized by the mean force $\langle f\rangle$ in each sample.

two point contacts, one would expect that such contacts should support on average a force two times the average force at side-vertex contacts. The discrepancy observed here means that the forces transmitted by side-side and side-vertex contacts are influenced by the overall structure of the packing although the nature (side-side or side-vertex) of the contacts prevails.

Figure 19 displays a map of contact forces for two extremal values of the polydispersity parameters $(s, \delta)$ together with the clusters of side-side contacts. As usual, the normal forces are represented by the thickness of the segments joining the particle centers. The visual impression that we get from these maps is consistent with the above picture of clusters elongated along strong force chains. Figure 19(b) provides a nice illustration of the multiscale nature of force distributions in highly polydisperse packings, as it was analyzed by Voivret et al. in the case of disk packings [51]. The average normal force $\left\langle f_{n}\right\rangle\left(d_{r}\right)$ as a function of the reduced size class $d_{r}$ is displayed in Fig. 20 for all values of $s$ and for $\delta=0$ and 1. The average force is a linear function of $d_{r}$ for all $s$, with an increasing slope as $s$ increases. Hence, the forces are captured by particles in proportion to their size. Moreover, the mean size class $d_{r}=0.5$ is the point of separation between the forces above and below the mean force.

\section{CONCLUSION}

In this paper, we presented a detailed numerical investigation of the combined effects of size polydispersity
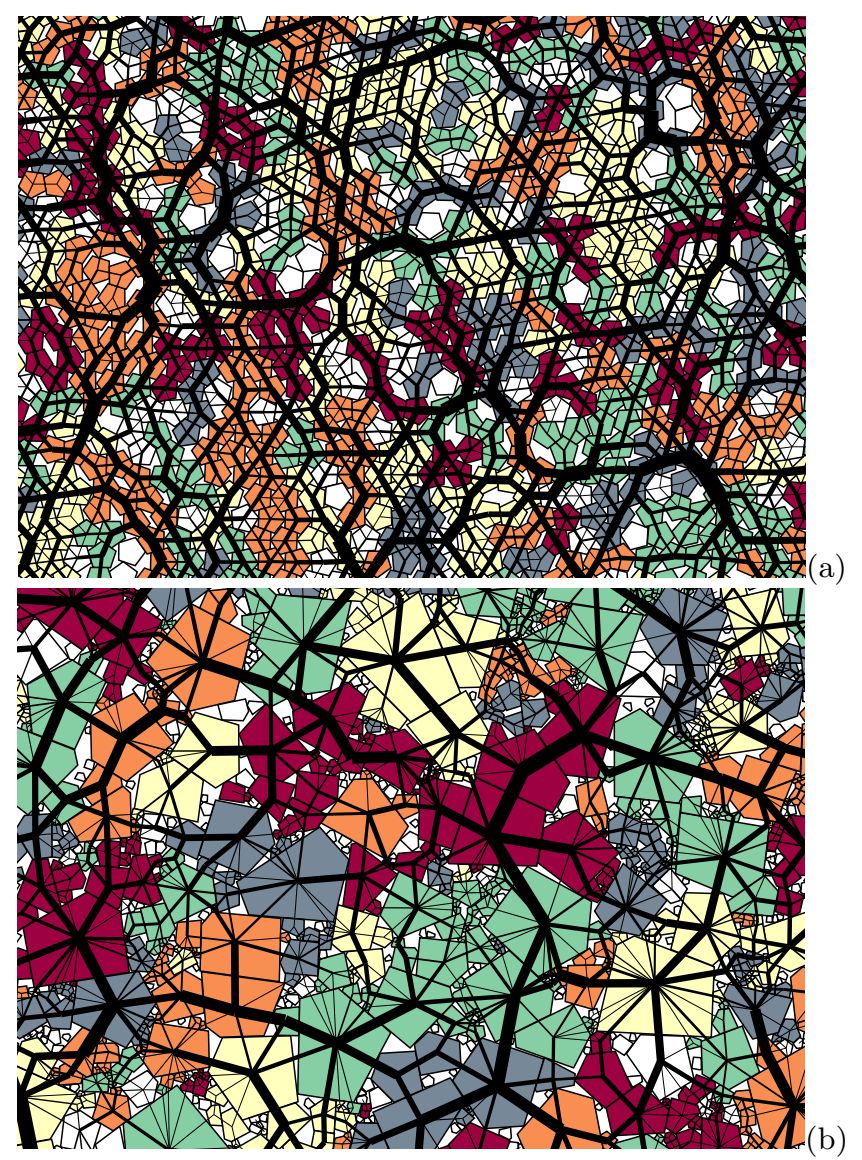

FIG. 19. (Color online) A snapshot of the contact force network for $(s, \delta)=(0.01,0)(\mathrm{a})$ and $(s, \delta)=(0.9,1)(\mathrm{b})$. The normal forces are represented by the thickness of the segments joining particle centers. The clusters of side-side contacts are also shown in different colors.

and particle shape polydispersity on the morphology and internal structures of 2D dense granular systems composed of frictionless pentagonal particles. The shape polydispersity was defined from the level of irregularity in the shape of pentagonal particles, quantified by a parameter $\delta$ varying from 0 (regular pentagon) to 1 (fully irregular pentagon). The particle size polydispersity was controlled by the size span $s$ varied from 0.01 (quasimonodisperse) to 0.9 (corresponding

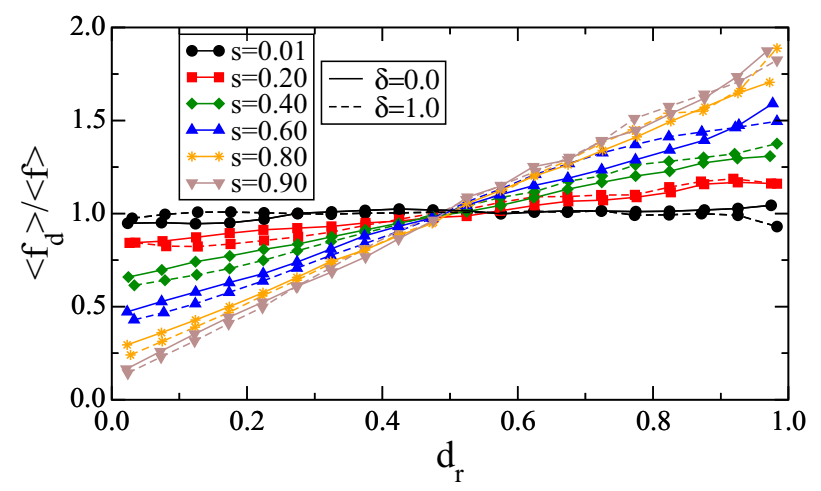

FIG. 20. (Color online) Mean force $\left\langle f_{d}\right\rangle$ as a function of reduced particle size $d_{r}$ for all values of $s$ and two values of $\delta$. 
to $d_{\max }=20 d_{\min }$ ) for a uniform size distribution by volume fractions. For each set of parameters $s$ and $\delta$, three independent packings composed of 5000 particles were prepared. The mean values, correlations, and fluctuations were analyzed for different values of $s$ and $\delta$ from the three independent data sets.

In the limit of monodisperse packings, we identified crystalline local structures characterized by positional and/or orientational ordering of pentagons. Despite local ordering and dense isostatic nature of the packings, the packing fraction is well below an ideally crystalline structure of pentagons best fitting to a triangular lattice and characterized by a packing fraction $\simeq 0.92$. The increase of packing fraction with $\delta$ was discussed as an effect of sharp corners intruding into the free volumes between particles.

An interesting finding of this work is to show clearly that the effect of shape polydispersity is significant only for a size span below $s=0.4$. This is true for all quantities analyzed in this paper such as the packing fraction, radial distribution functions, and clusters of side-side contacts. Below $s=0.4$, the size polydispersity is essentially a disorder parameter. In contrast, above $s=0.4$ the size distribution is broad enough for an increasing number of particles to fit into the pore space created by large particles. The value 0.4 is slightly below the approximative diameter of a disk fitting into the pore between four particles of unit diameter in a square arrangement. For $s>0.4$, the microstructural quantities reflect the interactions inside each size generation and with other size generations. In our packings, we have two generations for $0.4<s<0.7$ and three generations for $0.7<s<0.9$.

The role of side-side contacts was analyzed in detail with respect to the local structures. We showed that our pentagon packings are isostatic by accounting correctly for the kinematic constraints, each side-side contact representing two constraints. The proportion of side-side contacts is only marginally dependent on the polydispersity parameters, a property that should not be generalized to the packings of frictional pentagons where the connectivity of the particles is strongly dependent on the history of deformations undergone by the packing. The side-side contacts in our packings do not percolate but define clusters with an increasing number of particles as a function of $s$ and decreasing number of particles as a function of $\delta$. The clusters have anisotropic columnlike shapes with a decreasing aspect ratio as polydispersity increases. This feature was attributed to the effect of strong force chains by showing that the side-side contacts sustain larger forces than side-vertex contacts. Another important aspect of highly polydisperse packings is the multiscale transmission of forces. Each size class captures on average a mean force in proportion to its particle size. Moreover, the probability density of forces is increasingly broader as size polydispersity increases with a well-defined exponential fall-off of the number of forces.

A similar analysis is underway for frictional pentagon packings under steady shearing. The steady state in granular materials is a reference state in the sense that its rheological properties are independent of the strain history. The properties of frictionless pentagon packings are also almost independent of the packing preparation method due to their isostatic nature. For this reason, all the structural properties investigated in this paper reflect only the shape and size distributions of the particles. The steady state shearing of frictional pentagons is thus another instance where the effects of both size and shape polydispersity can be analyzed and compared to the present study with practically no reference to the preparation method. In particular, an important issue that needs to be clarified in this context is how the polydispersity parameters influence the shear strength.
[1] P. Acker, Statistical Models for Fracture in Disordered Media (North-Holland, Amsterdam, 1990), Chap. 3.2, pp. 52-58.

[2] F. de Larrard and T. Sedran, Cem. Concr. Res. 32, 1699 (2002).

[3] T. Aste and D. Weaire, The Pursuit of Perfect Packing (Institute of Physics Publishing, Bristol, 2000).

[4] H. J. Herrmann, R. M. Baram, and M. Wackenhut, Physica A (Amsterdam) 330, 77 (2003).

[5] Z.-Y. Li and Z.-Q. Zhang, Phys. Rev. B 62, 1516 (2000).

[6] N. B. Wilding, P. Sollich, and M. Buzzacchi, Phys. Rev. E 77, 011501 (2008).

[7] M. Allar and E. H. Sargent, Appl. Phys. Lett. 85, 5887 (2004).

[8] P. Sollich and N. B. Wilding, Phys. Rev. Lett. 104, 118302 (2010).

[9] E. Masoero, E. Del Gado, R. J.-M. Pellenq, F.-J. Ulm, and S. Yip, Phys. Rev. Lett. 109, 155503 (2012).

[10] S. Torquato and Y. Jiao, Phys. Rev. E 80, 041104 (2009).

[11] K. C. Smith, M. Alam, and T. Fisher, Phys. Rev. E 82, 051304 (2010).

[12] E. Azéma, N. Estrada, and F. Radjai, Phys. Rev. E 86, 041301 (2012).

[13] E. Azéma, F. Radjaï, and F. Dubois, Phys. Rev. E 87, 062203 (2013).
[14] A. Mirghasemi, L. Rothenburg, and E. Matyas, Geotechnique 52, 209 (2002).

[15] A. Donev, I. Cisse, D. Sachs, E. Variano, F. Stillinger, R. Connelly, S. Torquato, and P. Chaikin, Science 303, 990 (2004).

[16] A. Donev, R. Connelly, F. H. Stillinger, and S. Torquato, Phys. Rev. E 75, 051304 (2007).

[17] A. Donev, F. H. Stillinger, P. M. Chaikin, and S. Torquato, Phys. Rev. Lett. 92, 255506 (2004).

[18] E. Azéma and F. Radjaï, Phys. Rev. E 85, 031303 (2012).

[19] E. Azéma and F. Radjaï, Phys. Rev. E 81, 051304 (2010).

[20] C. R. A. Abreu, F. W. Tavares, and M. Castier, Powder Technol. 134, 167 (2003).

[21] A. Kyrylyuk and A. Philipse, Physica Status Solidi A 208, 2299 (2011).

[22] S. Sacanna, L. Rossi, A. Wouterse, and A. Philipse, J. Phys.: Condens. Matter 19, 376108 (2007).

[23] S. R. Williams and A. P. Philipse, Phys. Rev. E 67, 051301 (2003).

[24] A. Wouterse, S. R. Williams, and A. P. Philipse, J. Phys.: Condens. Matter 19, 406215 (2007).

[25] M. Boton, E. Azéma, N. Estrada, F. Radjaï, and A. Lizcano, Phys. Rev. E 87, 032206 (2013). 
[26] J. Zhao, S. Li, P. Lu, L. Meng, T. Li, and H. Zhu, Powder Technol. 214, 500 (2011).

[27] G. T. Nolan and P. E. Kavanagh, Powder Technol. 84, 199 (1995).

[28] B. Saint-Cyr, J.-Y. Delenne, C. Voivret, F. Radjaï, and P. Sornay, Phys. Rev. E 84, 041302 (2011).

[29] E. Azéma, F. Radjaï, B. Saint-Cyr, J.-Y. Delenne, and P. Sornay, Phys. Rev. E 87, 052205 (2013).

[30] Y. Jiao, F. H. Stillinger, and S. Torquato, Phys. Rev. E 81, 041304 (2010).

[31] M. Mailman, C. F. Schreck, C. S. O'Hern, and B. Chakraborty, Phys. Rev. Lett. 102, 255501 (2009).

[32] CeGeo, B. Saint-Cyr, K. Szarf, C. Voivret, E. Azéma, V. Richefeu, J.-Y. Delenne, G. Combe, C. Nouguier-Lehon, P. Villard, P. Sornay, M. Chaze, and F. Radjai, Europhys. Lett. 98, 44008 (2012).

[33] T. Aste, Phys. Rev. E 53, 2571 (1996).

[34] A. Bezrukov, M. Bargieł, and D. Stoyan, Part. Part. Syst. Charact. 19, 111 (2002).

[35] A. Kansal, S. Torquato, and F. Stillinger, J. Chem. Phys. 117, 8212 (2002).

[36] T.-S. Vu, G. Ovarlez, and X. Chateau, J. Rheol. 54, 815 (2010).

[37] R. Al-Raoush and M. Alsaleh, Powder Technol. 176, 47 (2007).

[38] E. Santiso and E. A. Müller, Mol. Phys. 100, 2461 (2002).

[39] R. S. Farr and R. D. Groot, J. Chem. Phys. 131, 244104 (2009).

[40] M. Hermes and M. Dijkstra, Europhys. Lett. 89, 38005 (2010).

[41] J. Dodds, J. Colloid Interface Sci. 77, 317 (1980).

[42] A. Clarke and J. Wiley, Phys. Rev. B 35, 7350 (1987).

[43] H. J. H. Brouwers, Phys. Rev. E 76, 041304 (2007).

[44] H. J. H. Brouwers, Phys. Rev. E 87, 032202 (2013).

[45] J. Mitchell and K. Soga, Fundamentals of Soil Behavior (Wiley, New York, 2005).

[46] L. Rothenburg and R. Bathurst, Geotechnique 39, 601 (1989).

[47] F. Radjai and S. Roux, The Physics of Granular Media, edited by H. Hinrichsen and D. E. Wolf (Wiley, Weinheim, 2005).

[48] F. Radjaï, J.-Y. Delenne, E. Azéma, and S. Roux, Granular Matter 14, 259 (2012).

[49] F. Radjaï and E. Azéma, Eur. J. Env. Civil Eng. 13, 203 (2009).

[50] C. Voivret, F. Radjaï, J.-Y. Delenne, and M. S. El Youssoufi, Phys. Rev. E 76, 021301 (2007).

[51] C. Voivret, F. Radjaï, J.-Y. Delenne, and M. S. El Youssoufi, Phys. Rev. Lett. 102, 178001 (2009).
[52] J. Moreau, Eur. J. Mech. A Solids 13, 93 (1994).

[53] M. Jean, Comput. Methods Appl. Mech. Eng. 177, 235 (1999).

[54] F. Radjaï and V. Richefeu, Mech. Mater. 41, 715 (2009).

[55] Discrete Numerical Modeling of Granular Materials, edited by F. Radjaï and F. Dubois (Wiley-ISTE, New-York, 2011).

[56] J. Moreau, Eur. J. Mech. A/Solids Suppl. 13, 93 (1994).

[57] F. Radjaï, M. Jean, J.-J. Moreau, and S. Roux, Phys. Rev. Lett. 77, 274 (1996)

[58] L. Staron, J.-P. Vilotte, and F. Radjaï, Phys. Rev. Lett. 89, 204302 (2002).

[59] A. Taboada, K. J. Chang, F. Radjaï, and F. Bouchette, J. Geophys. Res. 110, 1 (2005).

[60] M. Renouf and P. Alart, Comput. Methods Appl. Mech. Eng. 194, 2019 (2005).

[61] E. Azéma, F. Radjaï, R. Peyroux, F. Dubois, and G. Saussine, Phys. Rev. E 74, 031302 (2006).

[62] E. Azéma, F. Radjaï, R. Peyroux, V. Richefeu, and G. Saussine, Eur. Phys. J. E 26, 327 (2008).

[63] N. Estrada, A. Taboada, and F. Radjaï, Phys. Rev. E 78, 021301 (2008).

[64] N. Estrada, E. Azéma, F. Radjaï, and A. Taboada, Phys. Rev. E 84, 011306 (2011).

[65] V. Visseq, A. Martin, D. Iceta, E. Azema, D. Dureisseix, and P. Alart, Comput. Mech. 49, 709 (2012).

[66] F. Dubois, in Discrete Numerical Modeling of Granular Materials (Ref. [55]), pp. 233-262.

[67] G. Saussine, C. Cholet, P. Gautier, F. Dubois, C. Bohatier, and J. Moreau, Comput. Meth. Appl. Mech. Eng. 195, 2841 (2006).

[68] See Supplemental Material at http://link.aps.org/supplemental/ 10.1103/PhysRevE.90.012202 for video samples of the simulations analyzed in this paper.

[69] C. L. Henley, Phys. Rev. B 34, 797 (1986).

[70] A. Gervois and D. Bideau, in Disorder and Granular Media (Elsevier, Amsterdam, 1993), pp. 1-34.

[71] B. Saint-Cyr, J.-Y. Delenne, C. Voivret, F. Radjaï, and P. Sornay, Phys. Rev. E 84, 041302 (2011).

[72] H. Troadec, F. Radjaï, S. Roux, and J.-C. Charmet, Phys. Rev. E 66, 041305 (2002).

[73] J.-N. Roux, Phys. Rev. E 61, 6802 (2000).

[74] J. Baker and A. Kudrolli, Phys. Rev. E 82, 061304 (2010). 